\title{
ARTICLE
}

Animal Models

\section{Normalisation of circulating adiponectin levels in obese pregnant mice prevents cardiac dysfunction in adult offspring}

\author{
Owen R. Vaughan $\mathbb{1}^{1} \cdot$ Fredrick J. Rosario $^{1} \cdot$ Theresa L. Powell $^{1,2} \cdot$ Thomas Jansson $^{1}$
}

Received: 31 October 2018 / Revised: 6 March 2019 / Accepted: 26 March 2019 / Published online: 10 May 2019

(c) Springer Nature Limited 2019

\begin{abstract}
Background/objectives Adiponectin concentrations are low in obese pregnant women. Restoring normal adiponectin concentrations by infusion in obese pregnant mice prevents placental dysfunction, foetal overgrowth and metabolic syndrome in the offspring. We hypothesised that normalising maternal adiponectin in obese late pregnant dams prevents cardiac dysfunction in the adult offspring.

Subjects/methods Pregnant female mice with diet-induced obesity were infused with adiponectin $\left(0.62 \mu \mathrm{g} \mathrm{g}^{-1} \mathrm{day}^{-1}, n=\right.$ $24)$ or saline $(n=22)$ over days $14.5-18.5$ of pregnancy (term $=$ day 19.5). Control dams ate standard chow and received saline $(n=22)$. Offspring were studied at 3 and 6 months of age.

Results Maternal obesity impaired ventricular diastolic function, increased cardiomyocyte cross-sectional area and upregulated cardiac brain natriuretic peptide (Nppb) and $\alpha$-skeletal actin (Actal) gene expression in adult male offspring, compared to control offspring. In adult female offspring, maternal obesity increased $N p p b$ expression, decreased enddiastolic volume and caused age-dependent diastolic dysfunction but not cardiomyocyte hypertrophy. Maternal obesity also activated cardiac Akt and mechanistic target of rapamycin (mTOR) signalling in male, but not in female, offspring and inhibited cardiac extracellular signal-regulated kinase 1/2 (ERK1/2) in both sexes. Normalising maternal circulating adiponectin concentrations by infusing obese dams with adiponectin prevented offspring diastolic dysfunction and ventricular dilation and normalised cardiac Akt-mTOR signalling irrespective of sex. Maternal adiponectin infusion also reduced cardiac Nppb expression and increased ERK1/2 signalling in offspring of obese dams. Adiponectin infusion did not prevent cardiomyocyte hypertrophy but reduced ventricular wall thickness in male offspring and increased collagen content in female offspring of obese dams, compared to controls.

Conclusions Low maternal adiponectin levels in obese mice in late pregnancy are mechanistically linked to in utero programming of cardiac dysfunction in their offspring. Interventions enhancing endogenous adiponectin secretion or signalling in obese pregnant women could prevent the development of cardiac dysfunction in their children.
\end{abstract}

Supplementary information The online version of this article (https:// doi.org/10.1038/s41366-019-0374-4) contains supplementary material, which is available to authorized users.

Owen R. Vaughan

owen.vaughan@ucdenver.edu

1 Department of Obstetrics and Gynecology, University of Colorado Anschutz Medical Campus, Aurora, CO 80045, USA

2 Department of Pediatrics, University of Colorado Anschutz Medical Campus, Aurora, CO 80045, USA

\section{Introduction}

Over half of women now enter pregnancy overweight or obese [1,2]. Obesity in pregnancy programmes the infant for cardiometabolic disease in both childhood [3] and adult life $[4,5]$. Even before birth, high maternal pre-pregnancy body mass index is associated with impaired ventricular function and septal thickening in the first trimester foetus [6] and reduced diastolic function near term [7]. The risk for eccentric left ventricular hypertrophy at 6 years of age is also greater in the children of obese women $[8,9]$. Dietinduced obesity in pregnant experimental animals similarly induces offspring cardiac hypertrophy and impaired cardiac function both before and after birth [10-14]. Specifically, maternal obesity increases cardiac weight, wall thickness, 
cardiomyocyte cross-sectional area and collagen content in the foetus and adult offspring [14-16]. Adult offspring have enhanced ventricular expression of genes normally expressed in the foetal heart but characteristic of postnatal pathological hypertrophy, such as $\beta$ myosin heavy chain (Myh7), skeletal muscle alpha actin (Actal), atrial natriuretic peptide (Nppa) and brain natriuretic peptide (Nppb) [13, 14]. Cardiac protein kinase B (Akt), mechanistic target of rapamycin (mTOR), extracellular signalregulated kinase 1/2 (ERK1/2) and mitogen-activated protein kinase (MAPK) signalling pathways are also activated in offspring of obese dams [11, 13, 14]. These findings suggest that cardiomyocyte hypertrophy occurs in foetuses of obese mothers and is linked to the over-abundant supply of nutrients and elevated concentrations of insulin and other growth-promoting hormones in utero [11, 13, 14].

Obesity in pregnancy increases foetal nutrient supply and overgrowth in association with maternal insulin resistance, excessive activation of placental insulin and mTOR signalling and upregulation of specific placental nutrient transporters [17-20]. Circulating adiponectin concentrations are low in obese pregnant women [21, 22] and inversely related to birth weight $[21,23]$. Whereas adiponectin has insulin-sensitising effects in the skeletal muscle and liver, it inhibits placental insulin and mTOR signalling and placental amino acid transport [24-26]. When obese pregnant mice are infused with adiponectin to correct circulating concentrations to those in lean dams, placental dysfunction is prevented, and birth weight is normalised to control levels [27]. Offspring of obese dams infused with adiponectin exhibit normal postnatal growth and are themselves protected from developing obesity or metabolic disease [28]. These data demonstrate that low circulating adiponectin in obese dams is mechanistically linked to increased placental nutrient transport, foetal overnutrition, overgrowth and postnatal metabolic phenotype. We hypothesised that normalising maternal adiponectin levels in obese late pregnant dams also prevents cardiac dysfunction in the adult offspring. Because the effects of maternal obesity and foetal overgrowth on offspring metabolic phenotype differ with sex [29-32], we determined cardiac phenotype separately in male and female offspring of lean dams and obese dams, with or without adiponectin supplementation in late pregnancy.

\section{Materials and methods}

\section{Animals}

All procedures were approved by the Institutional Animal Care and Use Committee of the University of Colorado and conducted in accordance with NIH guidelines. Twelve- week-old C57/BL6J female mice (Jackson Laboratory, ME, USA), proven breeders, were randomly assigned to either a control diet (Con, D12489, Research Diets, New Brunswick, NJ, $10 \%$ calories from fat, $n=22$ ) or an obesogenic diet (Ob, Western Diet D12089B, Research Diets, 40\% calories from fat plus $20 \%$ sucrose solution ad lib, $n=46$ ), as previously described [19]. Females were mated with control males once animals in the Ob group had gained $25 \%$ of their initial body weight. At embryonic day (E) 14.5 (term $=\mathrm{E} 19.5)$, fasted dams were anaesthetised with isoflurane, and a miniosmotic pump was implanted subcutaneously [26, 27]. Dams on control diet received a continuous infusion of sterile phosphate-buffered saline (PBS). Obese animals were randomly allocated to receive either PBS $(n=22)$ or mouse recombinant full-length adiponectin from E14.5 through E18.5 (ADN, $n=24$, ALX522-059; Enzo Life Sciences, $0.62 \mu \mathrm{g}$ g of body weight ${ }^{-1}$ day $^{-1}$ ). In obese dams, this infusion rate increases circulating levels of adiponectin to those observed in normal weight dams [27]. Dams delivered spontaneously and were maintained on their respective diets throughout lactation. Offspring, once weaned at 28 days of age, were maintained on standard chow and housed in same-sex groups from multiple litters. Dams were euthanised by $\mathrm{CO}_{2}$ inhalation and cervical dislocation. One pup of each sex from each litter was used for each offspring outcome studied, therefore $n$ values represent individual litters. As a result of this design, three groups of offsprings were studied: offspring of dams on control diet infused with vehicle (Con/PBS), offspring of obese dams infused with vehicle (Ob/PBS), and offspring of obese dams with infusion of adiponectin $(\mathrm{Ob} /$ ADN). Sample sizes were based upon our previously published findings in this model [27, 28].

\section{Echocardiography}

Echocardiography was performed by a blinded operator to determine left ventricular morphology and systolic and diastolic function in a subset of offspring at age $\sim 90$ days (Con/PBS, $n=7$ males, 10 females; Ob/PBS, $n=7$ males, 6 females; Ob/ADN, $n=9$ males, 8 females). Serial transthoracic echocardiography and Doppler analyses were conducted under $2 \%$ isoflurane anaesthesia, with body temperature maintained at $37^{\circ} \mathrm{C}$, using the Vevo 2100 system (VisualSonics). Doppler measurements of mitral inflow and ventricular wall displacement at the level of the mitral valve annulus were used to determine the ratios of peak early and late diastolic inflow velocity $(E / A)$ and early and late diastolic tissue displacement $\left(E^{\prime} / A^{\prime}\right)$ as indices of cardiac diastolic function. Parasternal short axis images of the left ventricle at the level of the papillary muscle were collected in M-mode. Left ventricular wall thicknesses and internal diameters were used to calculate internal volume at 
A
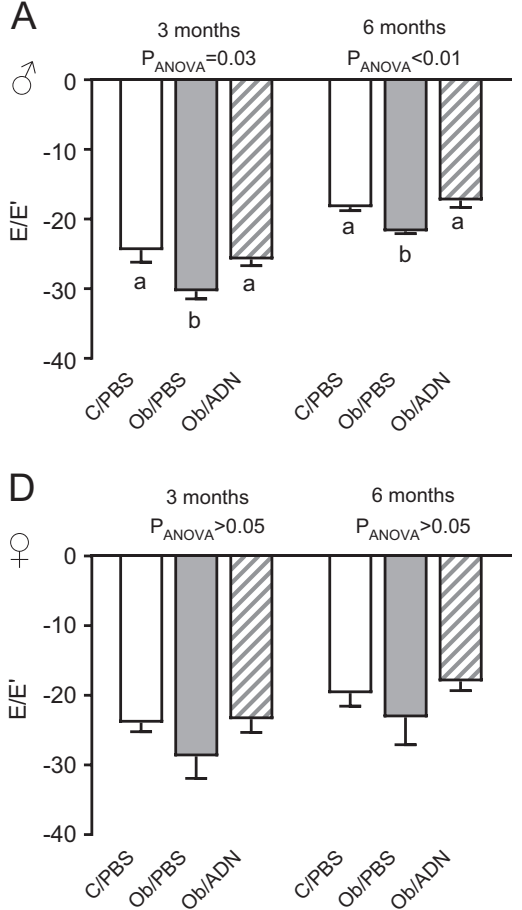

$\mathrm{B}$

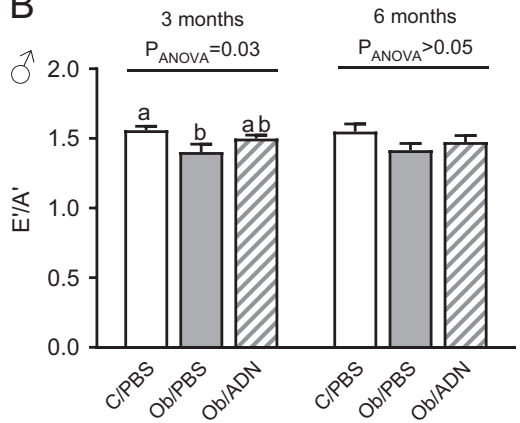

$E$

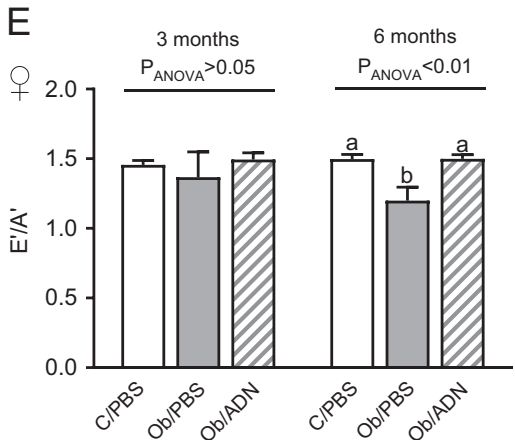

C

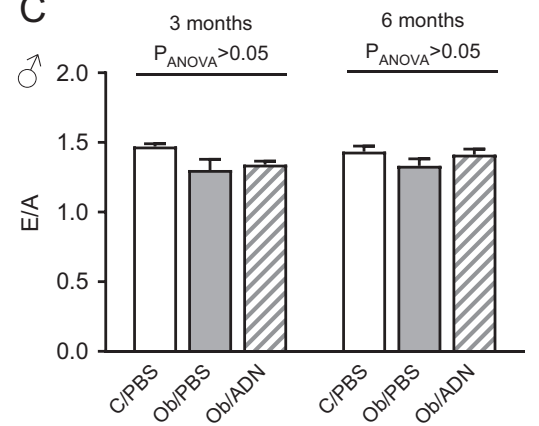

$\mathrm{F}$

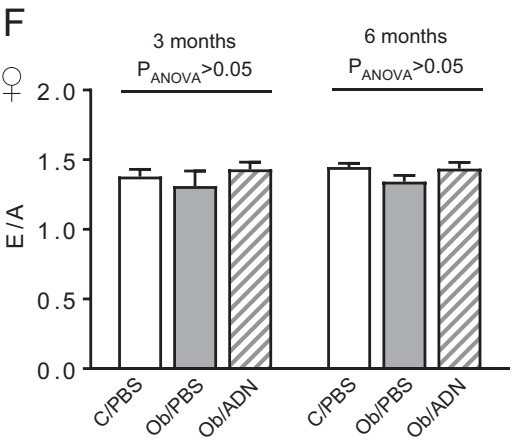

Fig. 1 Cardiac diastolic function in 3- and 6-month-old offspring. Mean \pm SEM echocardiographic indices of diastolic function in 3- and 6-month-old male $(n=7-9, \mathbf{a}-\mathbf{c})$ and female $(n=6-10, \mathbf{d}-\mathbf{f})$ offspring of control, phosphate-buffered saline (PBS)-infused pregnant mice $(\mathrm{C} /$ PBS) and obese pregnant mice infused either with PBS (Ob/PBS) or adiponectin (Ob/AND) from E14.5 to 18.5. $y$ Axes represent dimensionless ratios of velocities $\left(\mathrm{mm} \mathrm{s}^{-1}\right)$ of early-diastolic mitral inflow and ventricular wall displacement $\left(E / E^{\prime}\right)$, early- and late-diastolic ventricular wall displacement $\left(E^{\prime} / A^{\prime}\right)$ and early- and late-diastolic mitral inflow $(E / A)$. Overall effect of maternal treatment group, determined by one-way analysis of variance at each offspring age, given in figure. Values with different superscripts, a or b, are significantly different from one another by pairwise post hoc comparison $(P<0.05$, Holm-Sidak test $)$ systole and diastole using the leading-edge method, then ejection fraction (EF) and fractional shortening (FS) were determined as indices of cardiac systolic function. Measurements were averaged over at least four consecutive cycles. To determine the influence of age on cardiac function in offspring of obese dams, echocardiography was repeated in the same animals at 6 months of age, after which they were euthanised by $\mathrm{CO}_{2}$ asphyxiation and cervical dislocation. Hearts were perfused with $10 \% \mathrm{KCl}$, excised and weighed and a transverse slice was taken from the middle of the left ventricle and embedded in OCT. Remaining ventricular tissue was snap frozen in liquid $\mathrm{N}_{2}$. Offspring (Con/PBS, $n=12$ males, 8 females; Ob/PBS, $n=15$ males, 8 females; Ob/ADN, $n=15$ males, 11 females) that had not undergone echocardiography were euthanised at age 3 months without prior anaesthesia and the heart snap frozen immediately for signalling analyses.

\section{Histological analyses}

Embedded ventricular tissue was sectioned at $7 \mu \mathrm{m}$. Mounted, fixed sections were immunostained with Texas red-conjugated anti-wheat germ agglutinin antibody (1:2000, Thermo-Fisher Scientific) to delineate cardiomyocyte cell borders. Cardiomyocyte cross-sectional area was determined stereologically in at least 100 myocytes per sample [13]. Cardiac sections were also stained with picrosirius red to identify interstitial fibrillar collagen. Fibrosis was determined as the percentage area of tissue exhibiting birefringence under polarised light, using a set threshold across all samples.

\section{Gene expression}

Expression of type I collagen (Colla, Mm00801666_g1) and type III collagen genes (Col3a, Mm01254476_m1), the major fibrillar forms of collagen in the cardiac intersitium [33], was determined in ventricles of 3- and 6-month offspring using quantitative reverse transcriptase PCR and Taqman probes. Ventricular tissue from 3-month-old offspring was also used to determine the expression of the foetal cardiac gene programme: atrial natriuretic peptide (Nppa, Mm012555747_g1), brain natriuretic peptide (Nppb, Mm01255770_g1), $\beta$-myosin heavy chain (Myh7, Mm00600555_m1), $\alpha$-myosin heavy chain (Myh6, Mm00440359_m1), and skeletal muscle actin (Actal, Mm00808218_g1). Relative expression of the genes of interest was normalised to the geometric mean of $18 \mathrm{~S}$ 
Table 1 Cardiac morphology and systolic function in 3month-old offspring

\begin{tabular}{|c|c|c|c|c|}
\hline & Con/PBS & $\mathrm{Ob} / \mathrm{PBS}$ & $\mathrm{Ob} / \mathrm{ADN}$ & $P($ ANOVA $)$ \\
\hline Male offspring & $n=7$ & $n=7$ & $n=9$ & \\
\hline \multicolumn{5}{|l|}{ Left ventricle } \\
\hline Anterior wall (diastole), $\mathrm{mm}$ & $0.52 \pm 0.01$ & $0.51 \pm 0.01$ & $0.50 \pm 0.01$ & 0.34 \\
\hline Anterior wall (systole), mm & $0.73 \pm 0.01$ & $0.71 \pm 0.01$ & $0.71 \pm 0.01$ & 0.43 \\
\hline Posterior wall (diastole), $\mathrm{mm}$ & $0.52 \pm 0.01$ & $0.50 \pm 0.01$ & $0.50 \pm 0.01$ & 0.37 \\
\hline Posterior wall (systole), mm & $0.73 \pm 0.01$ & $0.71 \pm 0.01$ & $0.71 \pm 0.01$ & 0.31 \\
\hline Internal volume (diastole), $\mu \mathrm{l}$ & $57.3 \pm 2.8$ & $66.6 \pm 3.1$ & $64.1 \pm 3.1$ & 0.12 \\
\hline Internal volume (systole), $\mu$ l & $21.9 \pm 2.0$ & $25.6 \pm 2.4$ & $23.1 \pm 1.9$ & 0.49 \\
\hline Ejection fraction $(\%)$ & $62.2 \pm 1.8$ & $62.0 \pm 1.8$ & $64.3 \pm 1.8$ & 0.61 \\
\hline Fractional shortening (\%) & $33.0 \pm 1.2$ & $33.0 \pm 1.2$ & $34.7 \pm 1.4$ & 0.56 \\
\hline Heart rate, bpm & $502 \pm 14$ & $504 \pm 12$ & $532 \pm 11$ & 0.15 \\
\hline Female offspring & $n=10$ & $n=6$ & $n=8$ & \\
\hline \multicolumn{5}{|l|}{ Left ventricle } \\
\hline Anterior wall (diastole), $\mathrm{mm}$ & $0.51 \pm 0.01$ & $0.49 \pm 0.01$ & $0.48 \pm 0.01$ & 0.26 \\
\hline Anterior wall (systole), $\mathrm{mm}$ & $0.71 \pm 0.02$ & $0.69 \pm 0.01$ & $0.69 \pm 0.02$ & 0.53 \\
\hline Posterior wall (diastole), $\mathrm{mm}$ & $0.50 \pm 0.01$ & $0.48 \pm 0.01$ & $0.48 \pm 0.01$ & 0.25 \\
\hline Posterior wall (systole), $\mathrm{mm}$ & $0.71 \pm 0.01$ & $0.69 \pm 0.01$ & $0.69 \pm 0.02$ & 0.45 \\
\hline Internal volume (diastole), $\mu \mathrm{l}$ & $50.3 \pm 2.1^{\mathrm{a}}$ & $62.6 \pm 2.7^{b}$ & $55.6 \pm 3.4^{\mathrm{ab}}$ & 0.02 \\
\hline Internal volume (systole), $\mu$ l & $18.8 \pm 0.9$ & $23.5 \pm 2.3$ & $19.7 \pm 1.5$ & 0.10 \\
\hline Ejection fraction $(\%)$ & $62.6 \pm 1.1$ & $62.8 \pm 2.1$ & $64.6 \pm 1.5$ & 0.59 \\
\hline Fractional shortening (\%) & $33.1 \pm 0.8$ & $33.4 \pm 1.4$ & $34.6 \pm 1.1$ & 0.54 \\
\hline Heart rate, bpm & $542 \pm 13$ & $521 \pm 15$ & $529 \pm 6$ & 0.44 \\
\hline
\end{tabular}

Mean \pm SEM echocardiographic measurements of left ventricular morphology and systolic function and heart rate in 3-month-old offspring of control, PBS-infused pregnant mice and obese pregnant mice infused either with PBS or adiponectin from E14.5 to 18.5. Values in bold indicate significant overall effect of maternal treatment group by one-way ANOVA. Values with different superscripts, a or b, are significantly different from one another by pairwise post hoc comparison $(P<0.05$, Holm-Sidak test $)$

$A D N$ adiponectin, $A N O V A$ analysis of variance, $C o n$ control, $O b$ obese, $P B S$ phosphate-buffered saline ribosomal RNA (18S, Mm04277571_s1) and hypoxanthine phosphoribosyl transferase 1 (Hprt1, Mm00446968_m1) using the ddCt method. Endogenous reference gene expression was similar in the three experimental groups. Outlying relative expression values, identified using Grubbs' maximum normalised residual test with alpha = 0.01 , were omitted from subsequent statistical analysis but are reported in legend of Fig. 3.

\section{Protein abundance and phosphorylation}

Cardiac abundance of total and phosphorylated Akt, mTOR, ERK1/2, and p38 MAPK signalling proteins was determined using western blotting under reducing, denaturing conditions. Specific primary antibodies targeting phosphorylated (Thr308) and total Akt, phosphorylated (Ser235/ 236) and total S6 ribosomal protein (S6), phosphorylated (Thr37/46) and total eukaryotic translation initiation factor 4E-binding protein 1 (4EBP1), phosphorylated (Thr180/ Tyr182) and total p38 MAPK (P38MAPK) and phosphorylated (Thr202/Tyr204) and total p44/42 MAPK (ERK1/2) were obtained from Cell Signalling Technologies (Danvers, MA, USA; catalogue numbers \#4056, \#9272, \#2211, \#2217, \#9451, \#9459, \#9212, \#9216, \#9102, \#9101).

\section{Statistics}

Results are mean \pm SEM. The overall effect of maternal treatment was determined separately in male and female offspring, at each age, by one-way analysis of variance (ANOVA). When there was a significant overall effect of treatment on an outcome measurement, multiplicitycorrected pairwise comparisons were conducted between the three experimental groups using Holm-Sidak post hoc test. When the residuals of the data did not follow a normal distribution or variances differed between the experimental groups, data were $\log$ transformed prior to ANOVA, as indicated in the legends. Significance was accepted at the level $P<0.05$. 

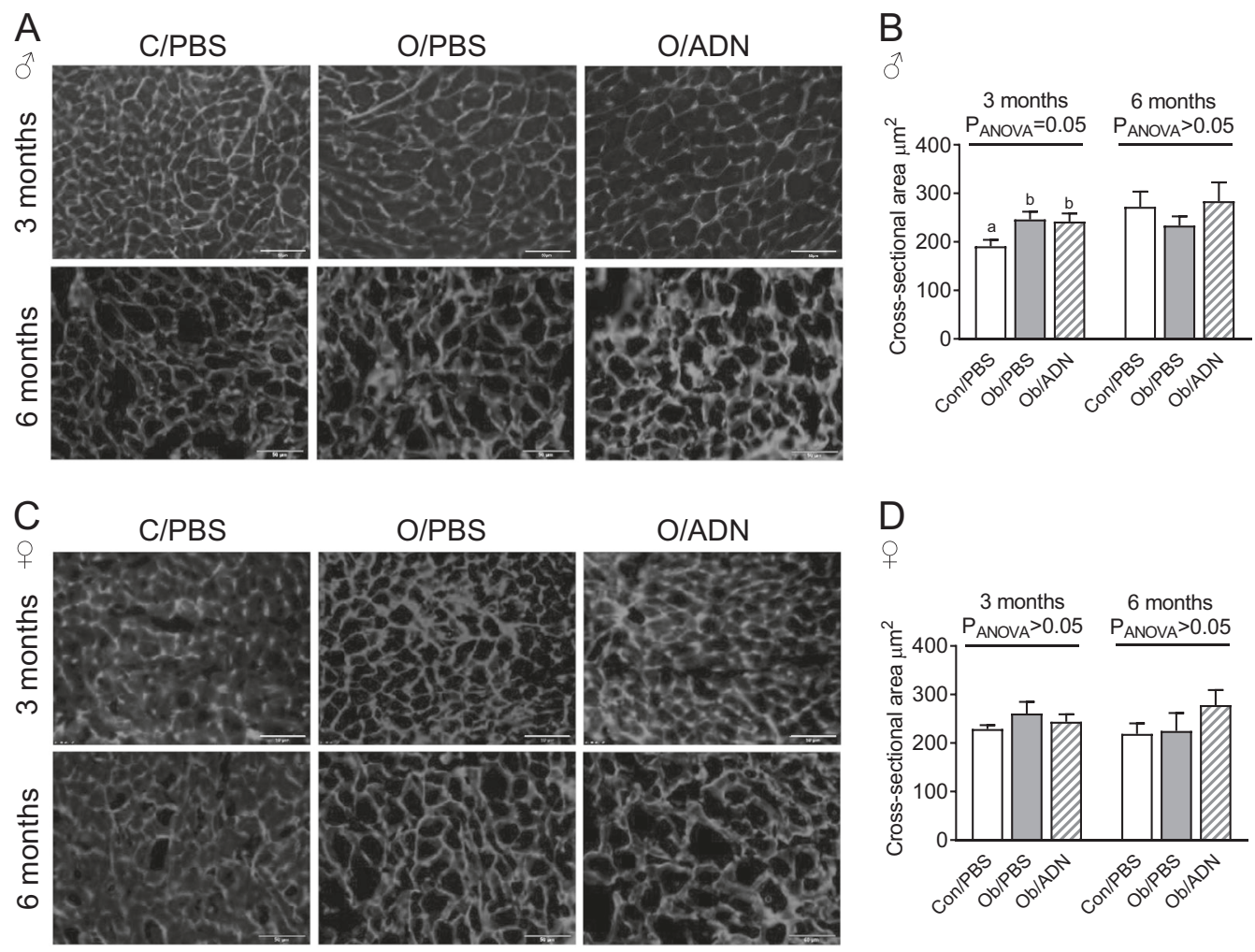

Fig. 2 Cardiomyocyte cross-sectional area in 3-month-old offspring. Representative images of wheat-germ agglutinin-stained transverse cardiac sections $(\mathbf{a}, \mathbf{c})$ and mean \pm SEM cardiomyocyte cross-sectional area in 3-month old male $(n=7-10, \mathbf{b})$ and female $(n=5-10, \mathbf{d})$ offspring of control, phosphate-buffered saline (PBS)-infused pregnant mice (C/PBS) and obese pregnant mice infused either with PBS $(\mathrm{Ob} /$

\section{Results}

\section{Cardiac structure and function}

Both adult male and female offspring of obese dams were heavier and had insulin-resistance and hyperinsulinaemia, compared to the offspring of lean dams [28]. Adiponectin supplementation in obese dams normalised offspring weight, insulin sensitivity and plasma insulin concentration to control levels at 3 months of age, as described previously [28]. In the present study, maternal obesity impaired cardiac diastolic function, measured either as reduced $E / E^{\prime}$ or $E^{\prime} / A^{\prime}$ ratio, in the male offspring (Fig. 1a, b) but not in the female offspring (Fig. 1d, e). Conversely, maternal obesity increased left ventricular end-diastolic volume in female but not in male offspring (Table 1). Adiponectin infusion in obese dams prevented diastolic dysfunction in the male offspring, such that $E / E$ ' and $E$ '/A' ratios were similar in Con/PBS and $\mathrm{Ob} /$ ADN animals (Fig. 1a, b), and attenuated end-diastolic volume towards control values in Ob/ADN female offspring (Table 1). There was no effect of maternal obesity or adiponectin infusion on left ventricular $E / A$ ratio, wall thickness or
PBS) or adiponectin (Ob/AND) from E14.5 to 18.5. Image scale bars represent $50 \mu \mathrm{M}$. Overall effect of maternal treatment group by oneway analysis of variance given in figure. Values with different superscripts, a or b, are significantly different from one another by pairwise post hoc comparison $(P<0.05$, Holm-Sidak test $)$

end-systolic volume in the 3-month-old offspring of C/PBS, $\mathrm{Ob} / \mathrm{PBS}$ and $\mathrm{Ob} / \mathrm{ADN}$ dams (Fig. 1 and Table 1).

When echocardiography was performed in the same animals at 6 months of age, the effects of maternal obesity and adiponectin on cardiac diastolic function were similar to those observed at 3 months (Fig. 1). Maternal obesity significantly reduced $E / E$ ' ratio in 6-month-old male offspring (Fig. 1a) and $E^{\prime} / A^{\prime}$ ratio in female offspring (Fig. 1f), and these changes were prevented by normalisation of maternal adiponectin levels during pregnancy. E/A ratio remained similar in all three groups of offspring, irrespective of sex. In contrast with the measurements at 3 months, left ventricular wall thickness was significantly reduced in male $\mathrm{Ob} /$ $\mathrm{ADN}$, but not in Ob/PBS, offspring compared to C/PBS animals, without any change in ventricular internal volume (Suppl. Table 1). Neither ventricular wall thickness nor internal volume differed between the three groups of female offspring at 6 months (Suppl. Table 1). Maternal obesity and adiponectin infusion did not affect offspring ventricular systolic function, expressed either as ejection fraction or fractional shortening, and heart rates during echocardiography were similar in all three offspring treatment groups, 
Table 2 Cardiac fibrosis in 3and 6-month-old offspring

\begin{tabular}{|c|c|c|c|c|}
\hline & Con/PBS & $\mathrm{Ob} / \mathrm{PBS}$ & $\mathrm{Ob} / \mathrm{ADN}$ & $P($ ANOVA $)$ \\
\hline \multicolumn{5}{|l|}{ Male offspring } \\
\hline 3-month collagen expression & $n=10$ & $n=14$ & $n=14$ & \\
\hline Colla1 & $1.00 \pm 0.34$ & $1.44 \pm 0.34$ & $1.56 \pm 0.33$ & 0.49 \\
\hline Col3al & $1.00 \pm 0.23$ & $1.52 \pm 0.28$ & $1.27 \pm 0.30$ & $0.76^{\mathrm{c}}$ \\
\hline 6-month collagen expression & $n=10$ & $n=10$ & $n=9$ & \\
\hline Collal & $1.00 \pm 0.15$ & $1.65 \pm 0.39$ & $0.91 \pm 0.14$ & 0.12 \\
\hline Col3a1 & $1.00 \pm 0.17$ & $1.67 \pm 0.40$ & $0.82 \pm 0.13$ & 0.08 \\
\hline Collagen content (picrosirius) & $n=6$ & $n=5$ & $n=10$ & \\
\hline$\%$ cross-sectional area & $5.2 \pm 1.2$ & $4.3 \pm 1.4$ & $2.9 \pm 0.6$ & 0.22 \\
\hline \multicolumn{5}{|l|}{ Female offspring } \\
\hline 3-month collagen expression & $n=7$ & $n=6$ & $n=10$ & \\
\hline Colla1 & $1.00 \pm 0.19$ & $0.79 \pm 0.19$ & $1.68 \pm 0.34$ & 0.07 \\
\hline Col3al & $1.00 \pm 0.08^{\mathrm{a}}$ & $1.16 \pm 0.18^{\mathrm{a}}$ & $2.10 \pm 0.32^{\mathrm{b}}$ & $<0.01$ \\
\hline 6-month collagen expression & $n=10$ & $n=10$ & $n=8$ & \\
\hline Collal & $1.00 \pm 0.08^{\mathrm{a}}$ & $1.43 \pm 0.25^{\mathrm{a}}$ & $2.63 \pm 0.55^{\mathrm{b}}$ & $<0.01^{\mathrm{c}}$ \\
\hline Col3al & $1.00 \pm 0.10^{\mathrm{a}}$ & $1.38 \pm 0.23^{\mathrm{ab}}$ & $1.87 \pm 0.30^{\mathrm{b}}$ & 0.03 \\
\hline Collagen content (picrosirius) & $n=8$ & $n=9$ & $n=7$ & \\
\hline$\%$ cross-sectional area & $2.6 \pm 0.3^{\mathrm{a}}$ & $3.7 \pm 0.8^{\mathrm{b}}$ & $6.3 \pm 0.8^{\mathrm{b}}$ & $<0.01$ \\
\hline
\end{tabular}

Mean \pm SEM relative expression of Collal and Col3al and collagen content determined by picrosirius staining in 3- and 6-month-old offspring of control, PBS-infused pregnant mice and obese pregnant mice infused either with PBS or adiponectin from E14.5 to 18.5. Values in bold indicate significant overall effect of maternal treatment group by one-way ANOVA. Values with different superscripts, a or b, are significantly different from one another by pairwise post hoc comparison $(P<0.05$, Holm-Sidak test $)$

$A D N$ adiponectin, $A N O V A$ analysis of variance, $C o n$ control, $O b$ obese, $P B S$ phosphate-buffered saline

'Indicates ANOVA performed on log transformed data. Statistical analysis of collagen content performed on combined 3- and 6-month old offspring irrespective of sex or postnatal age (Table 1 and Suppl. Table 1).

Following necropsy at age 6 months, absolute, but not relative, heart weight was greater in female Ob/PBS compared to Con/PBS offspring, while maternal adiponectin infusion corrected heart weight to control values (Suppl. Table 1). There was no effect of maternal obesity or adiponectin infusion on absolute or relative heart weight in 6month-old male offspring or on body weight in offspring of either sex. Maternal obesity increased cardiomyocyte crosssectional area by $30 \%$ in 3-month-old male offspring, irrespective of whether dams were infused with PBS or adiponectin (Fig. 2a, b), but did not affect cardiomyocyte cross-sectional area in 3-month-old female offspring (Fig. $2 \mathrm{c}, \mathrm{d})$. However, when cardiomyocyte cross-sectional areas were measured in $\mathrm{KCl}$-arrested hearts at age 6 months, they were similar in Con/PBS, OB/PBS and Ob/ADN offspring, irrespective of sex (Fig. 2).

\section{Cardiac foetal gene programme and collagen expression}

Neither Collal nor ColIIIal gene expression nor collagen content determined by picrosirius staining differed between the hearts of male offspring of the C/PBS, OB/PBS and OB/ ADN groups (Table 2). However, expression of Col3al was significantly greater in 3- and 6-month-old female offspring of obese, adiponectin-infused compared to control or obese, PBS-infused dams (Table 2). Similarly, the percentage cross-sectional area stained for collagen was greater in $\mathrm{Ob} /$ AND, compared to Con/PBS and Ob/PBS females, when 3and 6-month-old offspring were combined (Table 2). Collal expression was also increased in Ob/ADN female offspring at 6 months but not at 3 months.

Maternal obesity increased cardiac $N p p b$ gene expression in both male and female 3-month-old offspring, compared to the offspring of control dams (Fig. 3a, e). Adiponectin infusion in obese dams attenuated this upregulation, as evidenced by similar $N p p b$ expression in Con/PBS and $\mathrm{Ob} /$ ADN progeny. Maternal obesity similarly upregulated Actal expression in male offspring, and this effect was prevented by adiponectin supplementation (Fig. 3c), whereas Actal did not differ significantly between the three groups of female offspring (Fig. 3g). There was no significant effect of maternal treatment on cardiac Nppa expression in offspring of either sex (Fig. 3b, f).

The ratio of cardiac Myh7:Myh6 expression was significantly higher in female offspring of obese, PBS-infused 

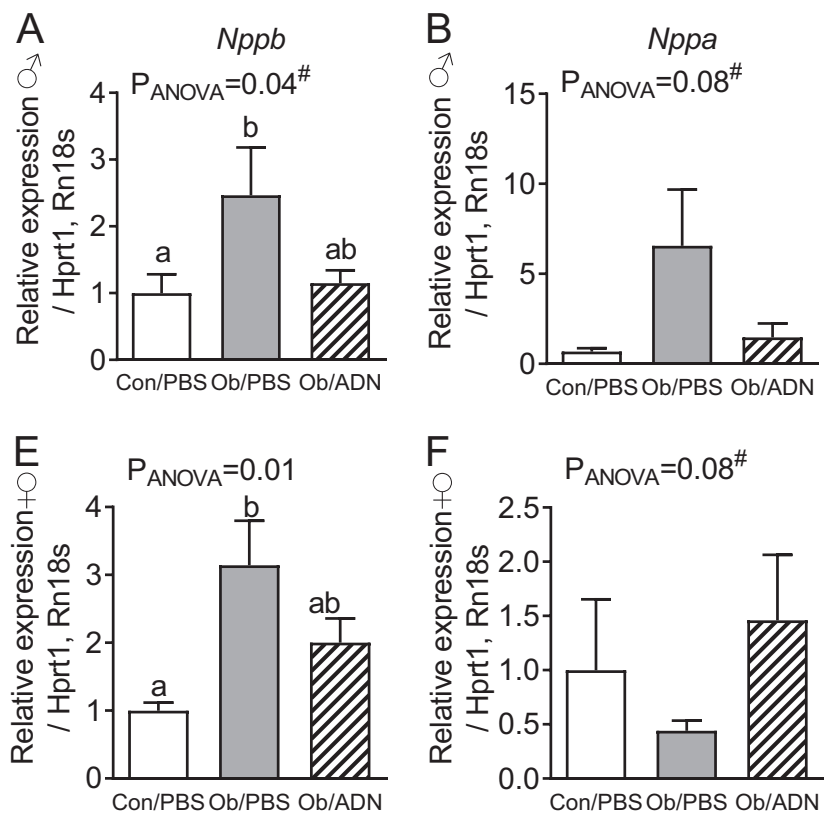

Fig. 3 Cardiac foetal gene programme expression in 3-month-old offspring. Mean \pm SEM relative expression of cardiac foetal genes indicative of pathological hypertrophy in the hearts of 3-month-old male (a-d, $n=12-15)$ and female (e-h, $n=8-11)$ offspring of control, phosphate-buffered saline (PBS)-infused pregnant mice (C/PBS) and obese pregnant mice infused either with PBS (Ob/PBS) or adiponectin (Ob/AND) from E14.5 to 18.5. Overall effect of maternal treatment group by one-way analysis of variance (ANOVA) given in

dams compared to lean, PBS-infused dams, whereas it was similar to control values in female Ob/ADN offspring (Fig. $3 \mathrm{~h})$. There was no effect of maternal treatment on female offspring heart expression of the individual genes $M y h 7$ (relative expression $\mathrm{C} / \mathrm{PBS} 1.00 \pm 0.12, \mathrm{Ob} / \mathrm{PBS} 1.80 \pm$ $0.42, \mathrm{Ob} / \mathrm{ADN} 1.75 \pm 0.35, P=0.20)$ and Myh6 (C/PBS $1.00 \pm 0.14, \mathrm{Ob} / \mathrm{PBS} \quad 0.78 \pm 0.16, \mathrm{Ob} / \mathrm{ADN} 1.48 \pm 0.32$, $P=0.13$ ). In males, there was no significant difference between treatment groups in either the ratio of Myh7:Myh6 (Fig. 3d) or the relative expression of $M y h 7$ (C/PBS $1.00 \pm$ 0.26 , Ob/PBS $2.04 \pm 0.76, \mathrm{Ob} / \mathrm{ADN} 1.29 \pm 0.42, P=0.40$ ) or Myh6 (C/PBS $1.00 \pm 0.16, \mathrm{Ob} / \mathrm{PBS} 1.03 \pm 0.13, \mathrm{Ob} / \mathrm{ADN}$ $1.34 \pm 0.31, P=0.21)$.

\section{Cardiac signalling}

Maternal obesity reduced total Akt abundance but increased threonine-308 phosphorylated Akt in the hearts of male offspring, compared to controls (Fig. 4a, b). Total cardiac S6 abundance was also reduced, while serine-236/236 phosphorylated S6 was increased in the male offspring of obese dams (Fig. 4a, b). Adiponectin supplementation in obese dams normalised cardiac abundance of total and phosphorylated Akt and S6. Adiponectin infusion also
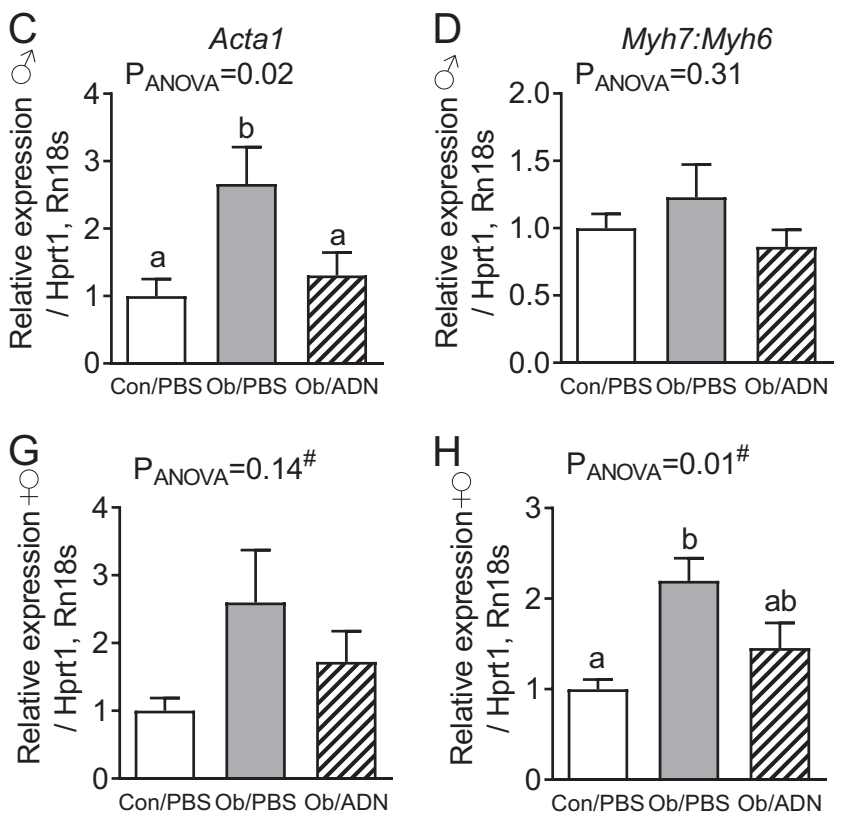

figure. Values with different superscripts, a or b, are significantly different from one another by pairwise post hoc comparison $(P<0.05$, Holm-Sidak test). Relative expression values of omitted outliers (Grubbs' test): a C/PBS 3.65, Ob/PBS 10.96; b C/PBS 4.53, Ob/PBS 0.45; c C/PBS 3.62, Ob/PBS 21.34; d Ob/PBS 38.22; e C/PBS 3.13, Ob/ADN 12.38; f Ob/ADN 28.43; h Ob/ADN 120.77. Hash (\#) indicates ANOVA performed on log-transformed data

reduced threonine-37/46 phosphorylated 4EBP1 in the male offspring of obese dams below levels in either the offspring of obese, PBS-infused dams or control dams, which were similar to each other (Fig. 4a, b). Total 4EBP1 abundance did not differ between the three groups. There was no effect of maternal obesity or adiponectin supplementation on the abundance of total or phosphorylated Akt, S6 and 4EBP1 in female offspring (Fig. 4a, c).

Maternal obesity reduced threonine-202/tyrosine-204 phosphorylation of ERK1/2 in both male and female offspring hearts and these changes were prevented by maternal adiponectin infusion, such that phosphorylated ERK1/2 abundance was similar to control levels in the Ob/ADN offspring (Fig. 4a-c). Total ERK1/2 was significantly reduced by maternal obesity in the female but not in male offspring, irrespective of maternal adiponectin infusion. Total abundance of p38 MAPK, but not threonine-180/ tyrosine-182 phosphorylated p38 MAPK, was significantly lower in the hearts of the Ob/ADN male offspring than in either C/PBS or Ob/PBS male offspring (Fig. 4a, b). Conversely, threonine-180/tyrosine-182 phosphorylation, but not total expression of p38 MAPK, was lower in female OB/ADN offspring, compared to female C/PBS and OB/ PBS animals (Fig. 4a, c). 
Fig. 4 Cardiac Akt, mammalian target of rapamycin, p38 mitogen-activated protein kinase (MAPK) and extracellular signal-regulated kinase $1 / 2$ (ERK1/2) signalling in 3-monthold offspring. a Representative bands from one western blot for each protein analysed. $\mathbf{b}, \mathbf{c}$ Mean \pm SEM relative abundance of total and phosphorylated forms of Akt, S6 ribosomal protein, 4EBP1, p38 MAPK and ERK1/2 proteins in the heart of 3-month-old male $(n=10-11$, b) and female $(n=7-8, \mathbf{c})$ offspring of control, phosphatebuffered saline (PBS)-infused pregnant mice (C/PBS) and obese pregnant mice infused either with PBS (Ob/PBS) or adiponectin (Ob/AND) from E14.5 to 18.5. Values in bold indicate significant overall effect of maternal treatment group by one-way analysis of variance.

Values with different superscripts, a or b, are significantly different from one another by pairwise post hoc comparison $(P<0.05$,

Holm-Sidak test)
A

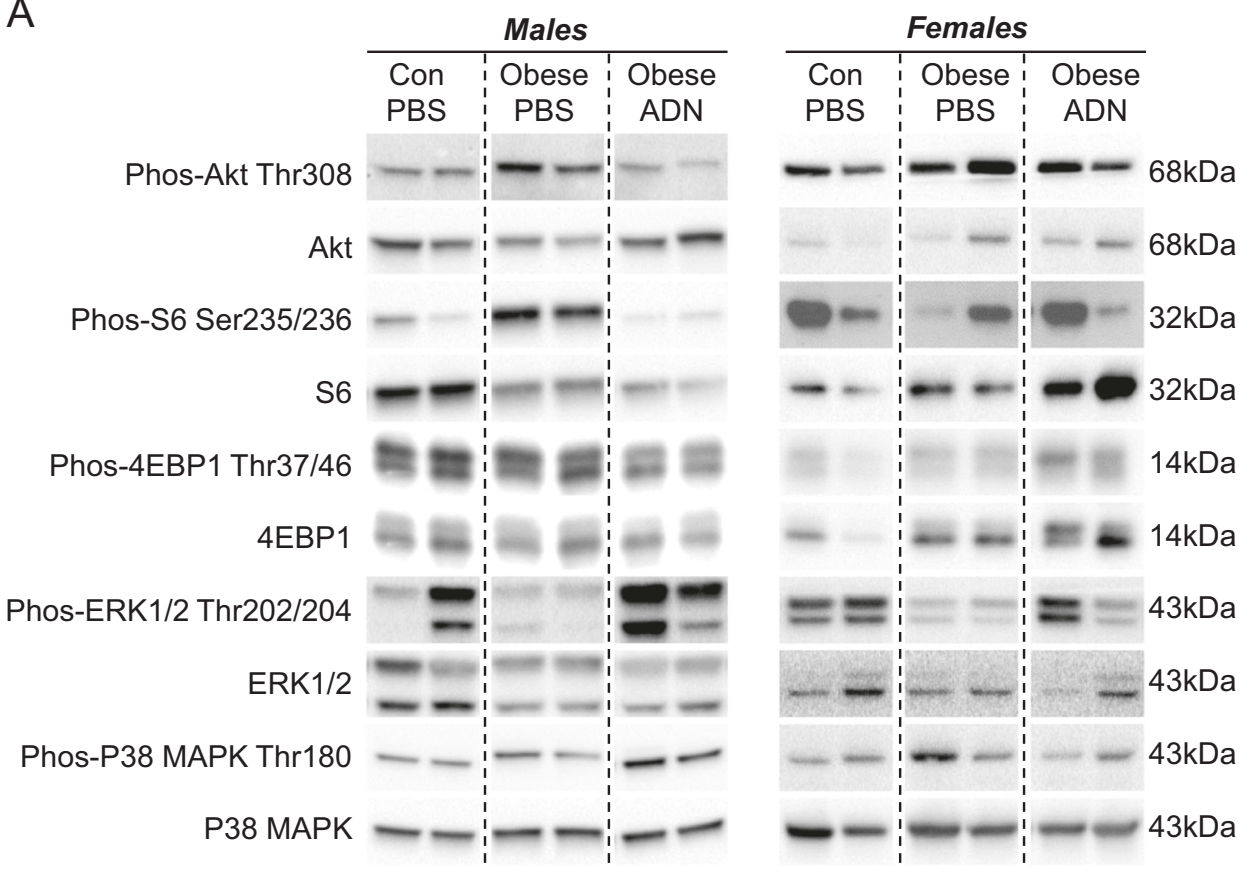

B

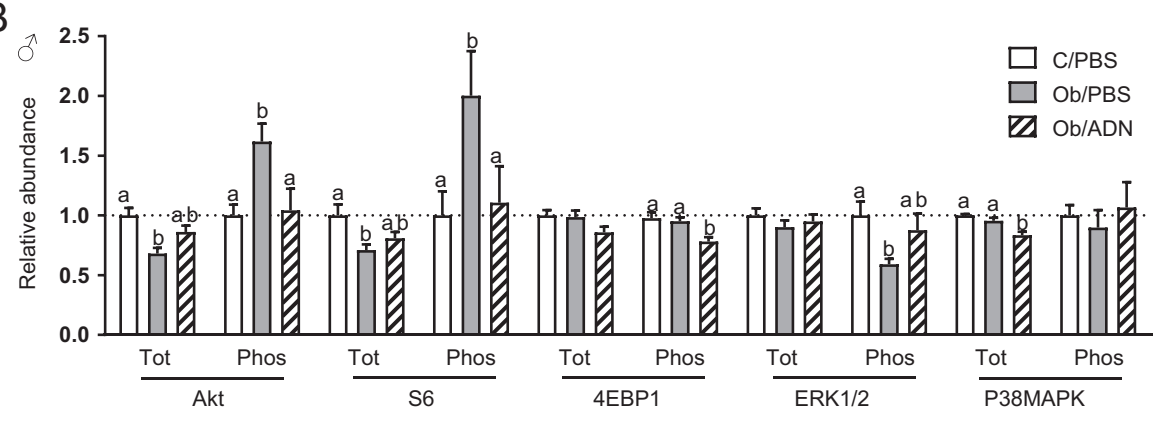

C

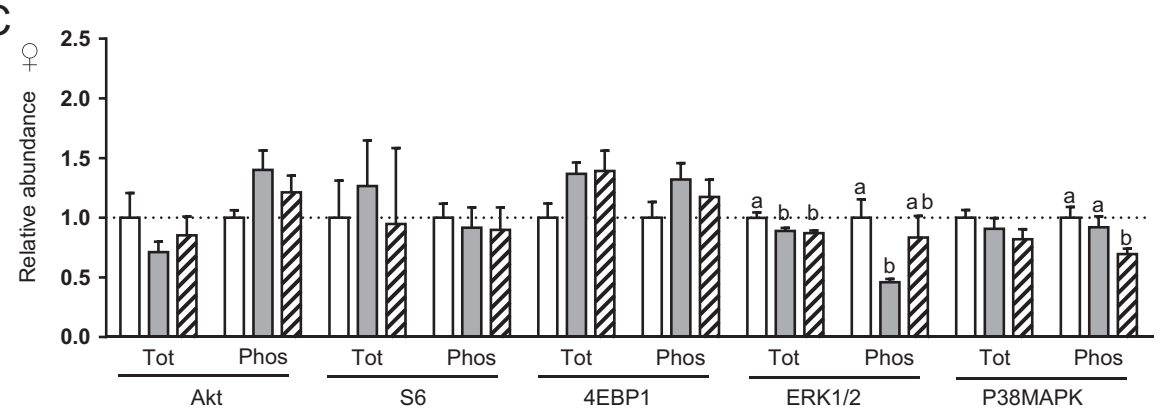

\section{Discussion}

This study is the first to show that maternal adiponectin supplementation in late pregnancy prevents cardiac dysfunction in the adult offspring of obese pregnant mice. Thus our findings support a mechanistic link between low adiponectin in pregnancy and programming of cardiovascular disease. We utilised a mouse model of maternal obesity in which obesity is induced prior to pregnancy by feeding a diet high in saturated fat, cholesterol and simple sugars [19], resembling the typical diet in developed countries. This model results in increased placental nutrient transport, foetal overgrowth and maternal metabolic alterations similar to those observed in obese pregnant women [19], contributing to the translational significance of our findings.

Using transthoracic echocardiography, we demonstrate that maternal obesity and foetal overgrowth are associated with left ventricular diastolic dysfunction in adulthood. Maternal obesity reduced tissue Doppler E'/A' ratio and $E /$ $E$ ' ratio in the male offspring, consistent with the lower 
diastolic relaxation rate in isolated hearts from male offspring of obese mice [13]. We found no evidence of systolic dysfunction in offspring of obese dams, in contrast with some previous animal studies showing reduced ventricular contractility both in vivo [34] and ex vivo [13]. Cardiac output may have been maintained by enhanced sympathetic tone in the offspring of obese dams under basal conditions [35] and we did not measure systolic function at high heart rates or during stress, when abnormal contraction is more marked in other animal models of maternal obesity [10, 13]. Similar to our study, left ventricular $E / A$ ratio and tissue Doppler $E$ '/A' ratio are reduced without systolic dysfunction both in rat neonates born to high-fat-fed dams [12] and third-trimester human foetuses of obese mothers [7]. Moreover, the $\sim 10 \%$ reduction in male offspring tissue Doppler $E$ '/A' ratio with maternal obesity in this study was quantitatively similar to that in human foetuses of obese mothers [7]. Taken together, the data therefore indicate that left ventricular diastolic dysfunction is a direct and persisting consequence of maternal obesity contributing to the developmental programming of cardiovascular morbidity in children of obese mothers.

Persisting cardiac dysfunction in the offspring of obese dams was independent of cardiomyocyte size, which was increased in male Ob/PBS offspring at 3 months but not at 6 months. In agreement with our results, previous longitudinal observations in a similar mouse model of maternal obesity show that marked cardiomyocyte hypertrophy in the juvenile offspring resolves before contractile dysfunction is observed at 12 weeks of age [13]. Diastolic dysfunction was also independent of interstitial collagen content, which was similar to control levels in the offspring of obese, PBSinfused dams. Maternal obesity induces cardiac fibrosis in the sheep foetus concomitant with inflammation and activated pro-fibrotic p38 MAPK signalling [16]. The absence of a difference in phosphorylated p38 MAPK abundance in Con/PBS and Ob/PBS offspring suggests that maternal obesity does not induce cardiac inflammation in our model. Maternal obesity may instead increase stiffness of the cardiomyocytes themselves. Certainly, in vitro relaxation is impaired in isolated cardiomyocytes from the offspring of pregnant mice fed a high-fat diet [36]. Greater expression of the foetal gene isoforms of the contractile proteins, $\alpha$-skeletal muscle actin (Actal) and $\beta$ myosin heavy chain (Myh7) may also have contributed to diastolic dysfunction in the offspring of obese dams by altering the calcium sensitivity and rate of sarcomeric cross-bridge cycling. Although the $\alpha$ to $\beta$-myosin heavy chain isoform switch appeared only as a trend in Ob/PBS males, previous rodent studies have shown that maternal obesity upregulates both cardiac $M y h 7$ and Actal gene expression [13, 14, 34] and that transgenic cardiac overexpression of $M y h 7$ exacerbates experimental cardiac dysfunction [37].
Cardiomyocyte hypertrophy and dysfunction were concomitant with increased cardiac Akt and mTOR signalling activity in male offspring of obese dams and therefore likely related to their peripheral insulin resistance and hyperinsulinaemia [28]. Increased cardiac Akt phosphorylation at threonine-308, downstream of the insulin receptor, has been reported previously in experimental animals exposed to maternal obesity [11, 14]. Moreover, both myocardial insulin signalling and pathological cardiac hypertrophy are correlated with plasma insulin concentrations in type 2 diabetics and in rats infused with insulin [38, 39]. Constitutive activation of cardiac Akt causes cardiomyocyte hypertrophy and increased foetal gene expression, whereas global Akt deletion attenuates cardiac dysfunction in rodents $[40,41]$. These signalling data therefore support the concept that cardiac dysfunction in the offspring of obese dams is a response to their programmed hyperinsulinaemia [28], linked to increased adiposity resulting from the overabundant supply of nutrients in utero. However, maternal obesity markedly reduced phosphorylation of ERK1/2, another downstream target of insulin signalling. Although maternal obesity increases cardiac ERK1/2 activity in other experimental animals [14, 39], genetic deletion of ERK1/2 exacerbates heart failure in hypertensive mice, suggesting that it serves a protective role in the heart [42]. Inhibition of cardiac ERK1/2 activation in the offspring of obese dams may therefore further contribute to cardiomyocyte dysfunction.

Although we did not measure offspring blood pressure, hypertension resulting from elevated peripheral vascular resistance may contribute to diastolic dysfunction in the offspring of obese mice [34, 43]. Indeed, increased ventricular expression of brain natriuretic peptide gene $(\mathrm{Nppb})$, which is normally induced by myocardial stretch, suggests increased cardiac haemodynamic load in these animals. Cardiac afterload may also be increased before birth in the foetuses of obese dams due to increased resistance in the placental vascular bed. Certainly, placental volume blood flow is reduced in pregnant macaques fed a high-fat diet, in association with placental inflammation [44]. However, whether maternal obesity results in foetal hypertension per se remains unknown in any species.

In contrast with the males, female offspring of obese dams did not exhibit diastolic dysfunction, cardiomyocyte hypertrophy or significant alterations in cardiac Akt and mTOR signalling at 3 months of age, despite similar upregulation of $N p p b$ and $M y h 7$ and suppression of ERK1/2 phosphorylation in both sexes. Females therefore appear initially protected from the adverse cardiac effects of maternal obesity. This sexual dimorphism reflects previous rodent studies showing more pronounced fat deposition, insulin resistance and hyperinsulinaemia in male than in female offspring of obese dams [29-32] including in the 3- 
month-old offspring of mice given the same dietary intervention as in this study [28]. Although there have been no previous sex-stratified studies of the effect of maternal obesity on offspring cardiac hypertrophy per se, dietinduced obesity in adult mice specifically increases left ventricular mass in males but not in females, in line with their respective insulin levels [45]. The more pronounced effect of maternal obesity in the hearts of male offspring therefore appears to be due to their more severely programmed metabolic phenotype. Higher levels of oestrogen and lower testosterone may also have been cardioprotective in female offspring, since oestrogen attenuates, whereas testosterone promotes cardiac dysfunction and hypertrophy $[46,47]$. Nevertheless, maternal obesity reduced diastolic $E$ '/A' ratio and increased absolute heart weight in 6-monthold female offspring, suggesting that any protective effect mediated by relative sex steroid levels was ultimately counterbalanced by their worsening metabolic phenotype with age.

Irrespective of sex or age, maternal adiponectin infusion prevented diastolic dysfunction and cardiac foetal gene upregulation in the offspring of obese dams. These findings are in line with our previous data showing that normalising maternal adiponectin concentrations inhibits excessive placental nutrient transport, foetal overgrowth, postnatal weight gain and insulin resistance associated with maternal obesity [19, 27, 28]. Therefore, reduced plasma insulin concentrations in adulthood most likely contributed to preventing cardiac dysfunction in male Ob/ADN offspring, as evidenced by the normalised phosphorylation of the insulin-responsive, pro-hypertrophic signalling molecules Akt and S6 in their hearts. The reduced cardiac abundance and phosphorylation of p38 MAPK in Ob/ADN male and female offspring, respectively, further suggests that maternal adiponectin alleviated offspring cardiac inflammation [16]. Maternal adiponectin may also have more directly prevented cardiac dysfunction in the offspring of obese dams by normalising nutrient delivery to the foetal heart in utero. Certainly, foetal cardiac hypertrophy in foetuses of obese pregnant sheep is associated with foetal hyperglycaemia [10], whereas maternal adiponectin infusion prevents foetal hyperglycaemia [27]. The effects of maternal exogenous adiponectin supplementation on foetal cardiac programming are unlikely to be mediated by directly increasing foetal circulating adiponectin, which circulates as a large multimeric peptide and cannot cross the placenta [48, 49]. Genetic manipulation of adiponectin expression in pregnant mice and their foetuses shows that its abundance in the foetal circulation is independent of a maternal source, even in obese dams [48]. The data therefore suggest that developmental programming of cardiac dysfunction is linked to low maternal adiponectin concentrations via elevated placental nutrient transport, which is common in obese pregnant women [17, 18, 21, 22].

Maternal adiponectin supplementation did not prevent the increase in cardiomyocyte size in the male offspring of obese dams, which occurred irrespective of infusion. However, cardiac hypertrophy resolved by 6 months and was not associated with dysfunction in these animals, and the ratio of Myh7:Myh6 expression remained similar to control levels. However, maternal adiponectin supplementation also caused a specific increase in cardiac fibrosis in female offspring and decreased wall thickness in male offspring, independent of obesity, indicating that there were some long-term adverse cardiac effects of the supplementation itself. Altering maternal adiponectin levels by infusion may affect cardiomyocyte development in utero, for example, by reducing foetal availability of mitogens such as insulin-like growth factor 1 [50], which normally stimulates foetal cardiomyocyte proliferation [51, 52]. Collagen gene activation and mild ventricular wall thinning in the offspring of adiponectin-supplemented dams might therefore be secondary to reduced cardiomyocyte endowment established in utero, although the specific developmental effects of the intervention remain unclear.

Overall, our data support the concept that offspring cardiac dysfunction is programmed in utero and mediated by the effects of low maternal adiponectin in obese dams. The design of our experiments does not allow us to assess the specific contributions of obesity during pregnancy vs. lactation to the programming of cardiac dysfunction. However, the fact that adiponectin supplementation during 4 days of late pregnancy prevented offspring cardiac dysfunction strongly suggests that cardiac programming occurs in utero. The results of this study also show that normalisation of maternal adiponectin concentrations in an animal model of maternal obesity with strong similarities to obese pregnant women largely prevents cardiac dysfunction in the offspring. We speculate that interventions enhancing endogenous adiponectin secretion or activating maternal adiponectin signalling could prevent the development of cardiac dysfunction in children of obese women.

Acknowledgements This work was supported by the National Institute of Health grants R24OD016724, R01HD065007 and 1S10OD018156-01. The authors are grateful to Kathryn Erickson, Kelsey Barner and Sara Wennersten for technical assistance.

\section{Compliance with ethical standards}

Conflict of interest The authors declare that they have no conflict of interest.

Publisher's note: Springer Nature remains neutral with regard to jurisdictional claims in published maps and institutional affiliations. 


\section{References}

1. Branum AM, Kirmeyer SE, Gregory EC. Prepregnancy body mass index by maternal characteristics and state: data from the birth certificate, 2014. Natl Vital Stat Rep. 2016;65:1-11.

2. Flegal KM, Kruszon-Moran D, Carroll MD, Fryar CD, Ogden CL. Trends in obesity among adults in the united states, 2005 to 2014. JAMA. 2016;315:2284-91.

3. Boney CM, Verma A, Tucker R, Vohr BR. Metabolic syndrome in childhood: association with birth weight, maternal obesity, and gestational diabetes mellitus. Pediatrics. 2005;115:e290-6.

4. Godfrey KM, Reynolds RM, Prescott SL, Nyirenda M, Jaddoe VWV, Eriksson JG, et al. Influence of maternal obesity on the long-term health of offspring. Lancet Diabetes Endocrinol. 2017;5:53-64.

5. Reynolds RM, Allan KM, Raja EA, Bhattacharya S, McNeill G, Hannaford PC, et al. Maternal obesity during pregnancy and premature mortality from cardiovascular event in adult offspring: follow-up of 1323275 person years. BMJ. 2013;347:f4539.

6. Ingul CB, Loras L, Tegnander E, Eik-Nes SH, Brantberg A. Maternal obesity affects fetal myocardial function as early as in the first trimester. Ultrasound Obstet Gynecol. 2016;47:433-42.

7. Ece I, Uner A, Balli S, Kibar AE, Oflaz MB, Kurdoglu M. The effects of pre-pregnancy obesity on fetal cardiac functions. Pediatr Cardiol. 2014;35:838-43.

8. Cade WT, Levy PT, Tinius RA, Patel MD, Choudhry S, Holland MR, et al. Markers of maternal and infant metabolism are associated with ventricular dysfunction in infants of obese women with type 2 diabetes. Pediatr Res. 2017;82:768-75.

9. Toemen L, Gishti O, van Osch-Gevers L, Steegers EA, Helbing WA, Felix JF, et al. Maternal obesity, gestational weight gain and childhood cardiac outcomes: role of childhood body mass index. Int J Obes (Lond). 2016;40:1070-8.

10. Wang J, Ma H, Tong C, Zhang H, Lawlis GB, Li Y, et al. Overnutrition and maternal obesity in sheep pregnancy alter the JNK-IRS-1 signaling cascades and cardiac function in the fetal heart. FASEB J. 2010;24:2066-76.

11. Fan X, Turdi S, Ford SP, Hua Y, Nijland MJ, Zhu M, et al. Influence of gestational overfeeding on cardiac morphometry and hypertrophic protein markers in fetal sheep. J Nutr Biochem. 2011;22:30-7.

12. Mdaki KS, Larsen TD, Wachal AL, Schimelpfenig MD, Weaver LJ, Dooyema SD, et al. Maternal high-fat diet impairs cardiac function in offspring of diabetic pregnancy through metabolic stress and mitochondrial dysfunction. Am J Physiol Heart Circ Physiol.2016;310:H681-92.

13. Blackmore HL, Niu Y, Fernandez-Twinn DS, Tarry-Adkins JL, Giussani DA, Ozanne SE. Maternal diet-induced obesity programs cardiovascular dysfunction in adult male mouse offspring independent of current body weight. Endocrinology. 2014;155:3970-80.

14. Fernandez-Twinn DS, Blackmore HL, Siggens L, Giussani DA, Cross CM, Foo R, et al. The programming of cardiac hypertrophy in the offspring by maternal obesity is associated with hyperinsulinemia, AKT, ERK, and mTOR activation. Endocrinology. 2012;153:5961-71.

15. Kandadi MR, Hua Y, Zhu M, Turdi S, Nathanielsz PW, Ford SP, et al. Influence of gestational overfeeding on myocardial proinflammatory mediators in fetal sheep heart. $\mathrm{J}$ Nutr Biochem. 2013;24:1982-90.

16. Huang Y, Yan X, Zhao JX, Zhu MJ, McCormick RJ, Ford SP, et al. Maternal obesity induces fibrosis in fetal myocardium of sheep. Am J Physiol Endocrinol Metab. 2010;299:E968-75.

17. Acosta O, Ramirez VI, Lager S, Gaccioli F, Dudley DJ, Powell TL, et al. Increased glucose and placental GLUT-1 in large infants of obese nondiabetic mothers. Am J Obstet Gynecol. 2015;212:227.e1-7.

18. Jansson N, Rosario FJ, Gaccioli F, Lager S, Jones HN, Roos S, et al. Activation of placental mTOR signaling and amino acid transporters in obese women giving birth to large babies. J Clin Endocrinol Metab. 2013;98:105-13.

19. Rosario FJ, Kanai Y, Powell TL, Jansson T. Increased placental nutrient transport in a novel mouse model of maternal obesity with fetal overgrowth. Obesity. 2015;23:1663-70.

20. Rosario FJ, Powell TL, Jansson T. Activation of placental insulin and mTOR signaling in a mouse model of maternal obesity associated with fetal overgrowth. Am J Physiol Regul Integr Comp Physiol. 2016;310:R87-93.

21. Jansson N, Nilsfelt A, Gellerstedt M, Wennergren M, HulthenRossander L, Powell TL, et al. Maternal hormones linking maternal bodymass index and dietary intake to birth weight. Am J Clin Nutr. 2008;87:1743-9.

22. Hendler I, Blackwell SC, Metha SH, Whitty JE, Russell E, Sorokin Y, et al. The levels of leptin, adiponectin, and resistin in normal weight, overweight, and obese pregnant women with and without preeclampsia. Am J Obstet Gynecol. 2005;193:979-83.

23. Segal P, Hamilton JK, Sermer M, Connelly PW, Hanley AJ, Zinman B, et al. Maternal obesity and familial history of diabetes have opposing effects on infant birth weight in women with mild glucose intolerance in pregnancy. J Matern Fetal Neonatal Med. 2008;21:73-9.

24. Jones HN, Jansson T, Powell TL. Full length adiponectin attenuates insulin signaling and inhibits insulin-stimulated amino acid transport in human primary trophoblast cells. Diabetes. 2010;59:1161-70.

25. ILMH Aye, Gao X, Weintraub ST, Jansson T, Powell TL. Inhibits insulin function in primary trophoblasts by PPAR $\alpha$-mediated ceramide synthesis. Mol Endocrinol2014;28:512-24.

26. Rosario FJ, Schumacher MA, Jiang J, Kanai Y, Powell TL, Jansson T. Chronic maternal infusion of full-length adiponectin in pregnant mice down-regulates placental amino acid transporter activity and expression and decreases fetal growth. J Physiol. 2012;590:1495-509.

27. Aye IL, Rosario FJ, Powell TL, Jansson T. Adiponectin supplementation in pregnant mice prevents the adverse effects of maternal obesity on placental function and fetal growth. Proc Natl Acad Sci USA. 2015;112:12858-63.

28. Paulsen ME, Rosario FJ, Wesolowski S, Powell T, Jansson T. Maternal adiponectin supplementation during pregnancy prevents adverse metabolic outcomes in offspring born to obese dams. FASEB J. 2019;33:2899-909.

29. Samuelsson AM, Matthews PA, Argenton M, Christie MR, McConnell JM, Jansen EH, et al. Diet-induced obesity in female mice leads to offspring hyperphagia, adiposity, hypertension, and insulin resistance: a novel murine model of developmental programming. Hypertension. 2008;51:383-92.

30. Yokomizo H, Inoguchi T, Sonoda N, Sakaki Y, Maeda Y, Inoue $\mathrm{T}$, et al. Maternal high-fat diet induces insulin resistance and deterioration of pancreatic beta-cell function in adult offspring with sex differences in mice. Am J Physiol Endocrinol Metab. 2014;306:E1163-75.

31. Ornellas F, Mello VS, Mandarim-de-Lacerda CA, Aguila MB. Sexual dimorphism in fat distribution and metabolic profile in mice offspring from diet-induced obese mothers. Life Sci. 2013;93:454-63.

32. Lecoutre S, Deracinois B, Laborie C, Eberle D, Guinez C, Panchenko PE, et al. Depot- and sex-specific effects of maternal obesity in offspring's adipose tissue. J Endocrinol. 2016;230:39-53.

33. Weber KT. Cardiac interstitium in health and disease: the fibrillar collagen network. J Am Coll Cardiol. 1989;13:1637-52. 
34. Loche E, Blackmore HL, Carpenter AAM, Beeson JH, Pinnock A, Ashmore TJ, et al. Maternal diet-induced obesity programmes cardiac dysfunction in male mice independently of post-weaning diet. Cardiovasc Res. 2018;114:1372-84.

35. Samuelsson A-M, Morris A, Igosheva N, L Kirk S, Pombo J, Coen $\mathrm{C}$, et al. Evidence for sympathetic origins of hypertension in juvenile offspring of obese rats. Hypertension. 2009;55:76-82.

36. Turdi S, Ge W, Hu N, Bradley KM, Wang X, Ren J. Interaction between maternal and postnatal high fat diet leads to a greater risk of myocardial dysfunction in offspring via enhanced lipotoxicity, IRS-1 serine phosphorylation and mitochondrial defects. J Mol Cell Cardiol. 2013;55:117-29.

37. Krenz M, Robbins J. Impact of beta-myosin heavy chain expression on cardiac function during stress. J Am Coll Cardiol. 2004;44:2390-7.

38. Cook SA, Varela-Carver A, Mongillo M, Kleinert C, Khan MT, Leccisotti L, et al. Abnormal myocardial insulin signalling in type 2 diabetes and left-ventricular dysfunction. Eur Heart J. 2010;31:100-11.

39. Samuelsson AM, Bollano E, Mobini R, Larsson BM, Omerovic E, $\mathrm{Fu} \mathrm{M}$, et al. Hyperinsulinemia: effect on cardiac mass/function, angiotensin II receptor expression, and insulin signaling pathways. Am J Physiol Heart Circ Physiol. 2006;291:H787-96.

40. Wende AR, O'Neill BT, Bugger H, Riehle C, Tuinei J, Buchanan $\mathrm{J}$, et al. Enhanced cardiac Akt/protein kinase B signaling contributes to pathological cardiac hypertrophy in part by impairing mitochondrial function via transcriptional repression of mitochondrion-targeted nuclear genes. Mol Cell Biol. 2015;35:831-46.

41. Shimizu I, Minamino T, Toko H, Okada S, Ikeda H, Yasuda N, et al. Excessive cardiac insulin signaling exacerbates systolic dysfunction induced by pressure overload in rodents. J Clin Invest. 2010;120:1506-14.

42. Purcell NH, Wilkins BJ, York A, Saba-El-Leil MK, Meloche S, Robbins J, et al. Genetic inhibition of cardiac ERK1/2 promotes stress-induced apoptosis and heart failure but has no effect on hypertrophy in vivo. Proc Natl Acad Sci USA. 2007;104:14074-9.
43. Samuelsson A-M, Matthews PA, Argenton M, Christie MR, McConnell JM, et al. Diet-induced obesity in female mice leads to offspring hyperphagia, adiposity, hypertension, and insulin resistance: a novel murine model of developmental programming. Hypertension2008;51:383-92.

44. Frias AE, Morgan TK, Evans AE, Rasanen J, Oh KY, Thornburg $\mathrm{KL}$, et al. Maternal high-fat diet disturbs uteroplacental hemodynamics and increases the frequency of stillbirth in a nonhuman primate model of excess nutrition. Endocrinology. 2011;152:2456-64.

45. Bohm C, Benz V, Clemenz M, Sprang C, Hoft B, Kintscher U, et al. Sexual dimorphism in obesity-mediated left ventricular hypertrophy. Am J Physiol Heart Circ Physiol. 2013;305:H211-8.

46. Wang YC, Xiao XL, Li N, Yang D, Xing Y, Huo R, et al. Oestrogen inhibits BMP4-induced BMP4 expression in cardiomyocytes: a potential mechanism of oestrogen-mediated protection against cardiac hypertrophy. Br J Pharmacol. 2015;172:5586-95.

47. Altamirano F, Oyarce C, Silva P, Toyos M, Wilson C, Lavandero $\mathrm{S}$, et al. Testosterone induces cardiomyocyte hypertrophy through mammalian target of rapamycin complex 1 pathway. J Endocrinol. 2009;202:299-307.

48. Qiao L, Yoo HS, Madon A, Kinney B, Hay WWJr, Shao J. Adiponectin enhances mouse fetal fat deposition. Diabetes2012;61:3199-207.

49. Mazaki-Tovi S, Kanety H, Pariente C, Hemi R, Efraty Y, Schiff E, et al. Determining the source of fetal adiponectin. J Reprod Med. 2007;52:774-8.

50. Qiao L, Wattez JS, Lee S, Guo Z, Schaack J, Hay WW Jr., et al. Knockout maternal adiponectin increases fetal growth in mice: potential role for trophoblast IGFBP-1. Diabetologia. 2016;59:2417-25.

51. Sundgren NC, Giraud GD, Schultz JM, Lasarev MR, Stork PJS, Thornburg KL. Extracellular signal-regulated kinase and phosphoinositol-3 kinase mediate IGF-1 induced proliferation of fetal sheep cardiomyocytes. Am J Physiol. 2003;285:R1481-9.

52. Kajstura J, Cheng W, Reiss K, Anversa P. The IGF-1-IGF-1 receptor system modulates myocyte proliferation but not myocyte cellular hypertrophy in vitro. Exp Cell Res. 1994;215:273-83. 